\title{
A Study on Farmer Response for Technical Intervention in Canal Command Area of Samrat Ashok Sagar Project
}

\author{
Shiv Singh Basediya*, S.K. Pyasi and R.N. Shrivastava \\ Department of Soil and Water Engineering, J. N. K. V. V., Jabalpur, India \\ *Corresponding author
}

\section{A B S T R A C T}

\section{Keywords}

Technical Intervention, Chi square test, Canal irrigation, Water

management, Water

productivity, Water user association

Article Info

Accepted:

26 February 2018

Available Online:

10 March 2018
Improved irrigation management and technical interventions proposed were discussed with farmers for getting their response. 15 farmers or water users out of the 4909 farmers of right bank canal command area was surveyed to discuss about technical interventions. A questionnaire was prepared considering the points made for improvement and information was collected through personal survey of individual farmer. To conduct this interview the study area was divided in three reaches that is head, middle and tail reach. In this way 180 farmers of all the five WUA were considered for analysis through Chi square test. It was found that average water productivity in case of sprinkler irrigation system, border irrigation system and flood irrigation system was recorded as $1.32 \mathrm{~kg} \mathrm{~m}-3,0.75 \mathrm{~kg} \mathrm{~m}-3$ and $0.61 \mathrm{~kg} \mathrm{~m}-3$ respectively. An increase of $76 \%$ in the water productivity was observed when border irrigation system was replaced by sprinkler irrigation system. Similarly $116 \%$ increase in water productivity was found when flood irrigation system was replaced by sprinkler irrigation system and about $23 \%$ increase in water productivity was found if flood irrigation system was replaced by border irrigation system.

\section{Introduction}

For improving irrigation water use efficiency and crop productivity through technological interventions, a clear understanding of the socio-economic condition of the farmers, present cropping system and constraints from the point of view of farmers is required. Among different stakeholders in irrigation system, farmers are the producers of agricultural outputs through the utilization of irrigation services provided to them. In-spite of being the most fundamental stakeholder, the farmers often receive the least attention for assessment of performance of water delivery system. It important to consider the issue of irrigation as a service provided to farmers. A set of criteria for constraints and performance evaluation of irrigation system needs to be considered from farmers point of view (Singh, 2003; Svendsen and Small, 1990; Ghosh et al., 2002).

We first discuss farmer response to scarcity under various scenarios of water distribution within an irrigation system. Second, we examine the theoretical responses of an individual farmer when faced with shortage or uncertainty, and hypothesize that the responses are distinct and conflicting. We then 
examine field data to test these hypotheses, and demonstrate that even very large irrigation systems, serving many hundreds of thousands of individual farmers, can be operated so as to induce a socially efficient response at the farm level to shortage and uncertainty, so that farmers' behavior is consistent with that which maximizes the benefit to society (Perry and Narayanamurthy, 1998).

The technical and economic feasibility of these interventions require studying the real field conditions. The modernization program of the canal irrigation system in Madhya Pradesh is going on in different commands with the help of various funding agencies as well as from national funds (Tanwar, 1998). The improvement and interventions to enhance the water use efficiency have been concentrated on renovating the canal network and not much emphasis was on below the outlet thus a need is being felt for identifying the interventions to be don below the out let level which will provide significant improvement in water use efficiency as well as water productivity the interventions design should not only improves water productivity but also should increase cropping intensity Rathod and Shah (2013). Which is important in improving the condition of farmers with this in view Samrat Ashok Sagar irrigation project of Vidisha district Madhya Pradesh is being selected as study area.

\section{Materials and Methods}

The study was conducted in the command area of Right Bank Canal of Samrat Ashok Sagar Irrigation Project located in Vidisha district, Madhya Pradesh (India). The Samrat Ashok Sagar Project is a major irrigation project located in Vidisha district of Madhya Pradesh (India). The dam is constructed on the Halaliriver, which is a tributary of Betwa River about $40 \mathrm{~km}$. from Bhopal. Command area of Samrat Ashok Sagar lies between
Longitude $77^{\circ} 33^{\prime} \mathrm{E}$ and Latitude $23^{\circ} 30^{\prime} \mathrm{N}$, at an altitude of $426 \mathrm{~m}$ respectively. The project is based on catchment and gravity flow. The problems of farmer at tail end canal command area, because optimum water is not available. However individual farmers use diesel and/or electric pump sets to lift water out of the canals. This project was commenced in year 1977 to irrigate 25091 hectares in Rabi season (Anonymous, 2016).

\section{Survey of the command area}

In order to assess the existing impact of canal irrigation on cropping pattern, cropping intensity, land use and agricultural production, farmers were contacted personally to collect the desired information in Performa. Representative farmers from WUA as well as from immediate vicinity were selected under different categories namely marginal, small, medium and large. Farmers were selected using stratified random sampling technique.

Three farmers in each of four categories were selected in head; middle and tail reach of RBC. Thus total 36 farmers were surveyed in each WUA area. The selected farmers were interviewed and the information on their agricultural practices, land use, crops grown, irrigation sources, irrigation practices were obtained.

Improved irrigation management and technical interventions proposed were discussed with farmers for getting their response. 71 farmers or water users out of the 87 farmers of command area of Ghatera Babaji tank canal were surveyed to discuss about technical interventions. A questionnaire was prepared considering the points made for improvement and information was collected through personal survey of individual farmer. The response of farmers was recorded in form of agreement or disagreement with the suggested improvement (Turkar, 2014). 


\section{Water users}

The right bank canal command area has 4909 farmers having total area 9503 ha. The land size of farmer's holdings varies between 0.2 ha to 20 ha. Details of water users, size of holding, water availability in different fields, crop grown, water resource and pumping facility in all three reaches i.e. head, middle and tail was collected through survey of farmers. The farmers were grouped into different categories as shown in table 1. Table 1 presents that maximum farmer's belong to marginal category having land holding of size 0.2 ha to 1 ha. There are 436 marginal holdings in head reach, 624 holdings in middle reach and 683 in tail reach.

\section{Results and Discussion}

Feasibility of surface irrigation and pressurized irrigation system was technically judged through an interview schedule and score matrix as suggested and adopted by Nema and Shrivastsva (2012). To conduct this interview the study area was divided in three reach that is head, middle and tail reach. Total 45 farmers were selected, surveyed and interviewed from each category of farmers. In this way 180 farmers of all the five WUA were considered for analysis through Chi square test. Information given by the farmers was compiled and the Chi-square test was applied. The contingency table of $(4 \times 4)$ regarding awareness of farmers about pressurized irrigation system was prepared under the farmer's category marginal, small, medium large and their response was recorded as nil, poor, medium and high. The Chi square test was applied as per the contingency table.

\section{Scoring matrix for characteristics of technology}

To study the characteristics of technology score matrix tables were prepared and the area was divided in to three reaches that is head, middle and tail reach. Total 36 farmers from each water user association were selected, surveyed and interviewed. Their response was obtained for each characters of technology and was recorded in table 2 to 10 (Shibiao et al., 2017; Pute et al., 2003; Wang et al., 2015; Pereira et al., 2012).

The tabulated data gives the scores given by the farmers of right bank canal command area of Samrat Ashok Sagar project Vidisha regarding the test of irrigation methods. The maximum score that could be awarded by a farmer was 10.

Sum of Square $(\mathrm{SS})=\left(\mathrm{T}_{1}^{2}+\mathrm{T}_{2}{ }^{2}+\mathrm{T}_{3}{ }^{2}\right) / \mathrm{r}-$ $\left(\mathrm{T}_{1}+\mathrm{T}_{2}+\mathrm{T}_{3}\right)^{2} / \mathrm{N}$

$\mathrm{SS}=\left(874^{2}+2303^{2}+2709^{2}\right) / 36-$ $(874+2303+2709)^{2} / 10=51612.05$

Chi square test $=12 \mathrm{x}(\mathrm{SS}) / \mathrm{N}(\mathrm{N}+1)=12 \mathrm{x}$ $51612.05 / 108(109)=52.62$

The Chi square test value was 52.62 which was larger than the tabulated value 5.99 and 9.21 on 2 degree of freedom at $5 \%$ and $1 \%$ level of significance. Thus the null hypothesis was rejected and concluded that there was significant difference among sprinkler irrigation, border irrigation and flood irrigation methods.

The Chi square test value was found 95.12 which was larger than the tabulated value 5.99 and 9.21 on 2 degree of freedom at $5 \%$ and $1 \%$ level of significance.

Thus the null hypothesis was rejected and concluded that there was significant difference among sprinkler irrigation, border irrigation and flood irrigation methods of irrigation as for as suitability for purchasing and cost involved in application of water through these methods. 

उ. सम्राट अशोक सागर परियोजना (हलाली), जिला : विदिशा / रायसेन
म.प्र. शासन जल संसाधन विभाग

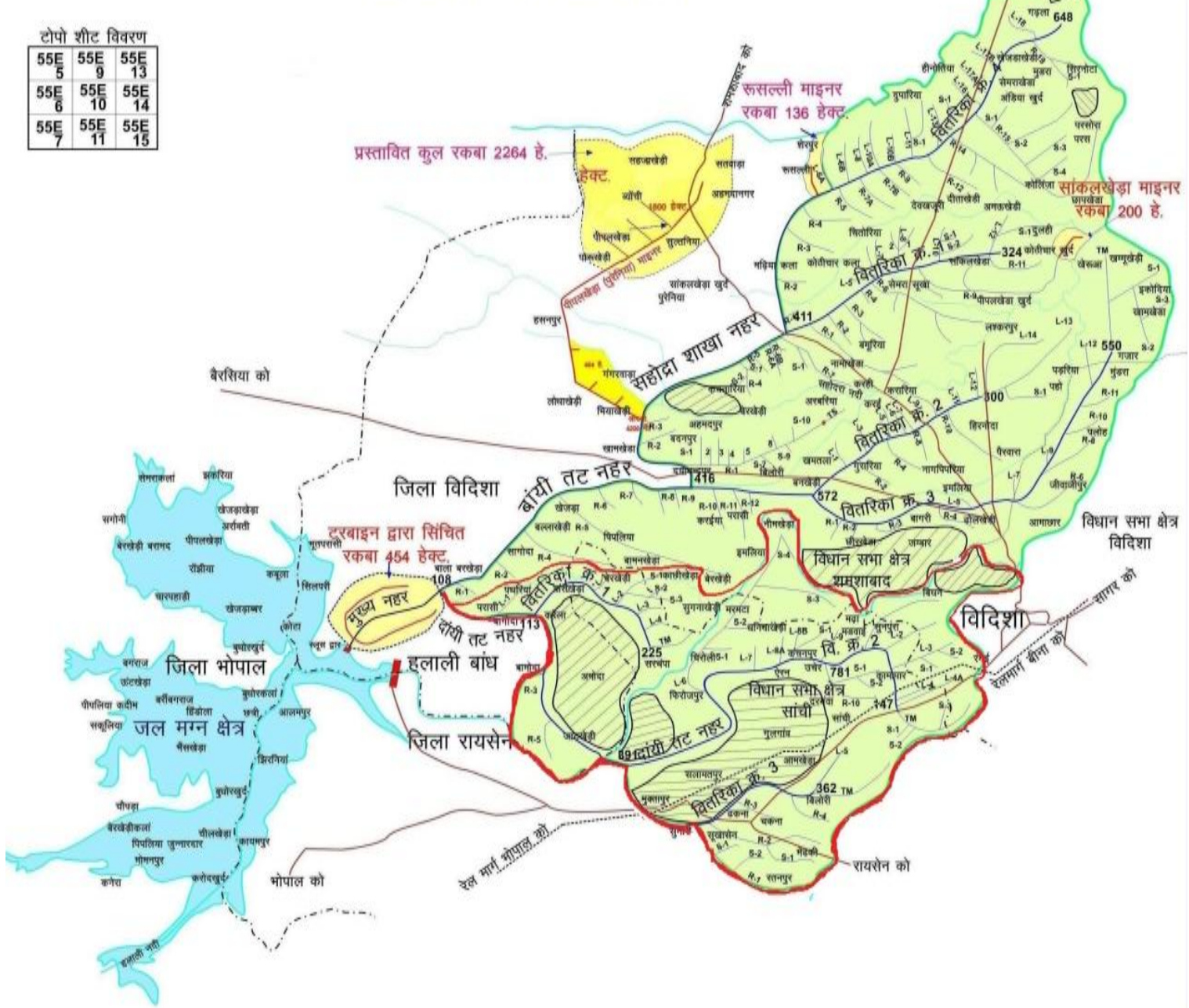

Table.1 Detail of water users in RBC command area

\begin{tabular}{|c|c|c|c|c|c|c|c|}
\hline \multirow[t]{2}{*}{ Farmers group } & \multicolumn{3}{|c|}{$\begin{array}{l}\text { Number of farmer's in } \\
\text { different reach }\end{array}$} & \multirow[t]{2}{*}{$\begin{array}{l}\text { Total } \\
\text { farmer }\end{array}$} & \multirow[t]{2}{*}{$\begin{array}{l}\% \text { of } \\
\text { farmers }\end{array}$} & \multirow[t]{2}{*}{$\begin{array}{l}\text { Area } \\
\text { (ha) }\end{array}$} & \multirow{2}{*}{$\begin{array}{l}\% \text { of } \\
\text { Area } \\
\text { (ha) }\end{array}$} \\
\hline & Head & Middle & Tail & & & & \\
\hline \multicolumn{8}{|c|}{ Land holding } \\
\hline $\begin{array}{l}\text { Marginal } \\
\text { farmers }\end{array}$ & 436 & 624 & 683 & 1743 & 36 & 1224 & 12.88 \\
\hline Small farmers & 422 & 613 & 511 & 1546 & 31 & 2554 & 26.88 \\
\hline $\begin{array}{l}\text { medium } \\
\text { farmers }\end{array}$ & 336 & 414 & 374 & 1124 & 23 & 3079 & 32.40 \\
\hline Large farmers & 164 & 155 & 177 & 496 & 10 & 2646 & 27.84 \\
\hline Total & 1358 & 1806 & 1745 & 4909 & 100 & 9503 & 100 \\
\hline
\end{tabular}


Table.2 Score matrix for labour requirement of different irrigation methods in Sayar WUA

\begin{tabular}{|c|c|c|c|c|c|c|c|c|c|}
\hline \multirow[t]{2}{*}{ Farmers name } & \multicolumn{3}{|c|}{$\begin{array}{l}\text { Marks given by the farmers, for } \\
\text { different Irrigation methods }\end{array}$} & \multicolumn{2}{|c|}{ Sprinkler } & \multicolumn{2}{|c|}{ Border } & \multicolumn{2}{|c|}{ Flood } \\
\hline & Sprinkler & Border & Flood & Obs & Rank & Obs & Rank & Obs & Rank \\
\hline Shakti singh & 7 & 3 & 5 & 2 & 13.5 & 1 & 4.5 & 3 & 27.5 \\
\hline Imaratsingh & 4 & 2 & 5 & 3 & 27.5 & 1 & 4.5 & 3 & 27.5 \\
\hline Gaindalal & 7 & 3 & 6 & 3 & 27.5 & 1 & 4.5 & 4 & 45.5 \\
\hline Ajabsingh & 5 & 4 & 6 & 3 & 27.5 & 1 & 4.5 & 4 & 45.5 \\
\hline Ganeshram & 8 & 1 & 5 & 4 & 45.5 & 1 & 4.5 & 4 & 45.5 \\
\hline Nandram & 7 & 3 & 6 & 4 & 45.5 & 1 & 4.5 & 4 & 45.5 \\
\hline Vishal singh & 7 & 5 & 3 & 4 & 45.5 & 1 & 4.5 & 4 & 45.5 \\
\hline Sangramsingh & 6 & 2 & 6 & 4 & 45.5 & 2 & 13.5 & 4 & 45.5 \\
\hline Tularam & 9 & 3 & 5 & 5 & 63.5 & 2 & 13.5 & 4 & 45.5 \\
\hline Dasharthsingh & 7 & 1 & 6 & 5 & 63.5 & 2 & 13.5 & 4 & 45.5 \\
\hline Deewansingh & 5 & 1 & 4 & 5 & 63.5 & 2 & 13.5 & 5 & 63.5 \\
\hline Harinarayan & 2 & 3 & 4 & 5 & 63.5 & 2 & 13.5 & 5 & 63.5 \\
\hline Bhairosingh & 8 & 3 & 7 & 5 & 63.5 & 2 & 13.5 & 5 & 63.5 \\
\hline Shyamlal & 9 & 1 & 5 & 6 & 79 & 2 & 13.5 & 5 & 63.5 \\
\hline Rupsingh & 5 & 3 & 5 & 6 & 79 & 2 & 13.5 & 5 & 63.5 \\
\hline Dhannalal & 7 & 5 & 7 & 6 & 79 & 3 & 27.5 & 5 & 63.5 \\
\hline Anarsingh & 8 & 2 & 5 & 7 & 92 & 3 & 27.5 & 5 & 63.5 \\
\hline Gajrajsingh & 4 & 3 & 4 & 7 & 92 & 3 & 27.5 & 5 & 63.5 \\
\hline Harprasad & 9 & 1 & 7 & 7 & 92 & 3 & 27.5 & 5 & 63.5 \\
\hline Maharajsingh & 3 & 3 & 4 & 7 & 92 & 3 & 27.5 & 5 & 63.5 \\
\hline Shakti singh & 8 & 4 & 4 & 2 & 13.5 & 1 & 4.5 & 3 & 27.5 \\
\hline Kamala bai & 4 & 4 & 6 & 7 & 92 & 3 & 27.5 & 5 & 63.5 \\
\hline Raghuveersingh & 8 & 3 & 5 & 7 & 92 & 3 & 27.5 & 6 & 79 \\
\hline Vipinsingh & 6 & 1 & 7 & 7 & 92 & 3 & 27.5 & 6 & 79 \\
\hline Ashok & 7 & 2 & 4 & 7 & 92 & 3 & 27.5 & 6 & 79 \\
\hline Bharat singh & 2 & 4 & 5 & 7 & 92 & 3 & 27.5 & 6 & 79 \\
\hline Baboolal & 8 & 2 & 3 & 8 & 101.5 & 3 & 27.5 & 6 & 79 \\
\hline Parsuram & 7 & 2 & 6 & 8 & 101.5 & 3 & 27.5 & 6 & 79 \\
\hline Bhagawansingh & 3 & 3 & 5 & 8 & 101.5 & 4 & 45.5 & 6 & 79 \\
\hline Hariram & 5 & 3 & 6 & 8 & 101.5 & 4 & 45.5 & 6 & 79 \\
\hline Bhairosingh & 9 & 2 & 3 & 8 & 101.5 & 4 & 45.5 & 6 & 79 \\
\hline Radheshyam & 3 & 4 & 4 & 8 & 101.5 & 4 & 45.5 & 6 & 79 \\
\hline Saitansingh & 4 & 2 & 6 & 9 & 106.5 & 4 & 45.5 & 7 & 92 \\
\hline Lakshminarayan & 6 & 1 & 5 & 9 & 106.5 & 4 & 45.5 & 7 & 92 \\
\hline Maharajsingh & 5 & 4 & 6 & 9 & 106.5 & 5 & 63.5 & 7 & 92 \\
\hline Jagannath & 7 & 1 & 4 & 9 & 106.5 & 5 & 63.5 & 7 & 92 \\
\hline Average & & - & & & 75.25 & - & 24.27 & - & 63.97 \\
\hline
\end{tabular}

T1- Sprinkler irrigation methods, T2- Border irrigation methods, T3- Flood irrigation methods and Obs- is Observations. 
Table.3 Scoring for cost in Sayar WUA

\begin{tabular}{|c|c|c|c|c|c|c|c|c|c|}
\hline \multirow[t]{2}{*}{ Farmers name } & \multicolumn{3}{|c|}{$\begin{array}{l}\text { Marks given by the farmers, } \\
\text { for different Irrigation } \\
\text { methods }\end{array}$} & \multicolumn{2}{|c|}{ Sprinkler } & \multicolumn{2}{|c|}{ Border } & \multicolumn{2}{|c|}{ Flood } \\
\hline & Sprinkler & $\begin{array}{l}\text { Borde } \\
\mathbf{r}\end{array}$ & Flood & Obs & Rank & Obs & Rank & Obs & Rank \\
\hline Shakti singh & 2 & 5 & 9 & 1 & 4 & 4 & 40.5 & 7 & 77 \\
\hline Imaratsingh & 3 & 5 & 8 & 1 & 4 & 4 & 40.5 & 7 & 77 \\
\hline Gaindalal & 3 & 5 & 9 & 1 & 4 & 4 & 40.5 & 7 & 77 \\
\hline Ajabsingh & 3 & 6 & 9 & 1 & 4 & 4 & 40.5 & 7 & 77 \\
\hline Ganeshram & 1 & 5 & 7 & 1 & 4 & 4 & 40.5 & 7 & 77 \\
\hline Nandram & 2 & 6 & 7 & 1 & 4 & 4 & 40.5 & 7 & 77 \\
\hline Vishal singh & 1 & 4 & 9 & 2 & 15 & 4 & 40.5 & 7 & 77 \\
\hline Sangramsingh & 3 & 4 & 9 & 2 & 15 & 5 & 50.50 & 7 & 77 \\
\hline Tularam & 1 & 6 & 9 & 2 & 15 & 5 & 50.50 & 8 & 88 \\
\hline Dasharthsingh & 3 & 5 & 7 & 2 & 15 & 5 & 50.50 & 8 & 88 \\
\hline Deewansingh & 2 & 6 & 8 & 2 & 15 & 5 & 50.50 & 8 & 88 \\
\hline Harinarayan & 3 & 6 & 9 & 2 & 15 & 5 & 50.50 & 8 & 88 \\
\hline Bhairosingh & 3 & 6 & 8 & 2 & 15 & 5 & 50.50 & 8 & 88 \\
\hline Shyamlal & 3 & 5 & 8 & 2 & 15 & 5 & 50.50 & 8 & 88 \\
\hline Rupsingh & 3 & 6 & 7 & 2 & 15 & 5 & 50.50 & 8 & 88 \\
\hline Dhannalal & 2 & 5 & 8 & 2 & 15 & 5 & 50.50 & 8 & 88 \\
\hline Anarsingh & 1 & 6 & 8 & 2 & 15 & 5 & 50.50 & 8 & 88 \\
\hline Gajrajsingh & 2 & 6 & 7 & 2 & 15 & 5 & 50.50 & 8 & 88 \\
\hline Harprasad & 2 & 5 & 9 & 2 & 15 & 5 & 50.50 & 8 & 88 \\
\hline Maharajsingh & 2 & 6 & 8 & 2 & 15 & 6 & 64.50 & 8 & 88 \\
\hline Shakti singh & 2 & 6 & 7 & 1 & 4 & 4 & 40.5 & 7 & 77 \\
\hline Kamala bai & 3 & 4 & 8 & 2 & 15 & 6 & 64.50 & 8 & 88 \\
\hline Raghuveersingh & 2 & 4 & 9 & 3 & 29.50 & 6 & 64.50 & 9 & 101.50 \\
\hline Vipinsingh & 3 & 6 & 7 & 3 & 29.50 & 6 & 64.50 & 9 & 101.50 \\
\hline Ashok & 3 & 4 & 8 & 3 & 29.50 & 6 & 64.50 & 9 & 101.50 \\
\hline Bharat singh & 2 & 4 & 8 & 3 & 29.50 & 6 & 64.50 & 9 & 101.50 \\
\hline Baboolal & 3 & 5 & 8 & 3 & 29.50 & 6 & 64.50 & 9 & 101.50 \\
\hline Parsuram & 2 & 4 & 9 & 3 & 29.50 & 6 & 64.50 & 9 & 101.50 \\
\hline Bhagawansingh & 3 & 5 & 9 & 3 & 29.50 & 6 & 64.50 & 9 & 101.50 \\
\hline Hariram & 2 & 6 & 9 & 3 & 29.50 & 6 & 64.50 & 9 & 101.50 \\
\hline Bhairosingh & 2 & 6 & 8 & 3 & 29.50 & 6 & 64.50 & 9 & 101.50 \\
\hline Radheshyam & 1 & 6 & 7 & 3 & 29.50 & 6 & 64.50 & 9 & 101.50 \\
\hline Saitansingh & 2 & 4 & 7 & 3 & 29.50 & 6 & 64.50 & 9 & 101.50 \\
\hline $\begin{array}{l}\text { Lakshminaraya } \\
\text { n }\end{array}$ & 1 & 5 & 8 & 3 & 29.50 & 6 & 64.50 & 9 & 101.50 \\
\hline Maharajsingh & 1 & 6 & 9 & 3 & 29.50 & 6 & 64.50 & 9 & 101.50 \\
\hline Jagannath & 2 & 5 & 9 & 3 & 29.50 & 6 & 64.50 & 9 & 101.50 \\
\hline Average & & - & & - & 18.50 & - & 54.50 & - & 90.50 \\
\hline
\end{tabular}


Table.4 Scoring for suitability of irrigation method Sayar WUA

\begin{tabular}{|c|c|c|c|c|c|c|c|c|c|}
\hline \multirow[t]{2}{*}{ Farmers name } & \multicolumn{3}{|c|}{$\begin{array}{l}\text { Marks given by the farmers, } \\
\text { for different Irrigation } \\
\text { methods }\end{array}$} & \multicolumn{2}{|c|}{ Sprinkler } & \multicolumn{2}{|c|}{ Border } & \multicolumn{2}{|c|}{ Flood } \\
\hline & Sprinkler & Border & Flood & Obs & Rank & Obs & Rank & Obs & Rank \\
\hline Shakti singh & 8 & 7 & 5 & 6 & 60 & 4 & 30.5 & 2 & 5 \\
\hline Imaratsingh & 8 & 4 & 4 & 6 & 60 & 4 & 30.5 & 2 & 5 \\
\hline Gaindalal & 6 & 6 & 3 & 6 & 60 & 4 & 30.5 & 2 & 5 \\
\hline Ajabsingh & 9 & 5 & 2 & 6 & 60 & 4 & 30.5 & 2 & 5 \\
\hline Ganeshram & 7 & 4 & 4 & 6 & 60 & 4 & 30.5 & 2 & 5 \\
\hline Nandram & 8 & 6 & 3 & 7 & 79 & 5 & 45 & 2 & 5 \\
\hline Vishal singh & 8 & 6 & 5 & 7 & 79 & 5 & 45 & 2 & 5 \\
\hline Sangramsingh & 6 & 7 & 5 & 7 & 79 & 5 & 45 & 2 & 5 \\
\hline Tularam & 9 & 4 & 2 & 7 & 79 & 5 & 45 & 3 & 16.5 \\
\hline Dasharthsingh & 7 & 7 & 3 & 7 & 79 & 5 & 45 & 3 & 16.5 \\
\hline Deewansingh & 7 & 5 & 3 & 7 & 79 & 5 & 45 & 3 & 16.5 \\
\hline Harinarayan & 8 & 5 & 2 & 7 & 79 & 5 & 45 & 3 & 16.5 \\
\hline Bhairosingh & 9 & 6 & 4 & 7 & 79 & 5 & 45 & 3 & 16.5 \\
\hline Shyamlal & 6 & 4 & 5 & 7 & 79 & 5 & 45 & 3 & 16.5 \\
\hline Rupsingh & 8 & 7 & 3 & 7 & 79 & 5 & 45 & 3 & 16.5 \\
\hline Dhannalal & 7 & 6 & 2 & 7 & 79 & 6 & 60 & 3 & 16.5 \\
\hline Anarsingh & 7 & 6 & 4 & 7 & 79 & 6 & 60 & 3 & 16.5 \\
\hline Gajrajsingh & 9 & 5 & 4 & 8 & 96.5 & 6 & 60 & 3 & 16.5 \\
\hline Harprasad & 6 & 5 & 3 & 8 & 96.5 & 6 & 60 & 3 & 16.5 \\
\hline Maharajsingh & 8 & 7 & 2 & 8 & 96.5 & 6 & 60 & 3 & 16.5 \\
\hline Shakti singh & 7 & 5 & 2 & 6 & 60 & 4 & 30.5 & 2 & 5 \\
\hline Kamala bai & 8 & 7 & 4 & 8 & 96.5 & 6 & 60 & 3 & 16.5 \\
\hline Raghuveersingh & 7 & 4 & 3 & 8 & 96.5 & 6 & 60 & 3 & 16.5 \\
\hline Vipinsingh & 9 & 6 & 2 & 8 & 96.5 & 6 & 60 & 4 & 30.5 \\
\hline Ashok & 7 & 5 & 3 & 8 & 96.5 & 6 & 60 & 4 & 30.5 \\
\hline Bharat singh & 8 & 5 & 3 & 8 & 96.5 & 7 & 79 & 4 & 30.5 \\
\hline Baboolal & 6 & 6 & 2 & 8 & 96.5 & 7 & 79 & 4 & 30.5 \\
\hline Parsuram & 7 & 7 & 4 & 8 & 96.5 & 7 & 79 & 4 & 30.5 \\
\hline Bhagawansingh & 9 & 7 & 3 & 8 & 96.5 & 7 & 79 & 4 & 30.5 \\
\hline Hariram & 7 & 4 & 4 & 8 & 96.5 & 7 & 79 & 4 & 30.5 \\
\hline Bhairosingh & 8 & 7 & 3 & 9 & 105.5 & 7 & 79 & 4 & 30.5 \\
\hline Radheshyam & 6 & 5 & 3 & 9 & 105.5 & 7 & 79 & 5 & 45 \\
\hline Saitansingh & 7 & 6 & 2 & 9 & 105.5 & 7 & 79 & 5 & 45 \\
\hline Lakshminarayan & 7 & 5 & 3 & 9 & 105.5 & 7 & 79 & 5 & 45 \\
\hline Maharajsingh & 8 & 7 & 5 & 9 & 105.5 & 7 & 79 & 5 & 45 \\
\hline Jagannath & 8 & 7 & 3 & 9 & 105.5 & 7 & 79 & 5 & 45 \\
\hline Average & - & & & - & 86.08 & - & 56.72 & - & 20.69 \\
\hline
\end{tabular}


Table.5 Scoring for control and management of water in command area in Sayar WUA

\begin{tabular}{|c|c|c|c|c|c|c|c|c|c|}
\hline \multirow[t]{2}{*}{ Farmers name } & \multicolumn{3}{|c|}{$\begin{array}{l}\text { Marks given by the farmers, } \\
\text { for different Irrigation } \\
\text { methods }\end{array}$} & \multicolumn{2}{|c|}{ Sprinkler } & \multicolumn{2}{|c|}{ Border } & \multicolumn{2}{|c|}{ Flood } \\
\hline & Sprinkler & Border & Flood & Obs & Rank & Obs & Rank & Obs & Rank \\
\hline Shakti singh & 7 & 6 & 4 & 6 & 61.5 & 32 & 34 & 1 & 4 \\
\hline Imaratsingh & 9 & 6 & 3 & 6 & 61.5 & 32 & 36 & 1 & 4 \\
\hline Gaindalal & 7 & 5 & 1 & 6 & 61.5 & 32 & 37 & 1 & 4 \\
\hline Ajabsingh & 8 & 5 & 4 & 6 & 61.5 & 5 & 45.5 & 1 & 4 \\
\hline Ganeshram & 8 & 7 & 5 & 6 & 61.5 & 5 & 45.5 & 1 & 4 \\
\hline Nandram & 9 & 6 & 1 & 7 & 79 & 5 & 45.5 & 1 & 4 \\
\hline Vishal singh & 7 & 4 & 2 & 7 & 79 & 5 & 45.5 & 2 & 12 \\
\hline Sangramsingh & 9 & 5 & 2 & 7 & 79 & 5 & 45.5 & 2 & 12 \\
\hline Tularam & 8 & 7 & 4 & 7 & 79 & 5 & 45.5 & 2 & 12 \\
\hline Dasharthsingh & 8 & 5 & 3 & 7 & 79 & 5 & 45.5 & 2 & 12 \\
\hline Deewansingh & 6 & 6 & 3 & 7 & 79 & 5 & 45.5 & 2 & 12 \\
\hline Harinarayan & 9 & 7 & 1 & 7 & 79 & 5 & 45.5 & 2 & 12 \\
\hline Bhairosingh & 7 & 6 & 1 & 7 & 79 & 5 & 45.5 & 2 & 12 \\
\hline Shyamlal & 8 & 7 & 4 & 7 & 79 & 5 & 45.5 & 2 & 12 \\
\hline Rupsingh & 6 & 4 & 4 & 7 & 79 & 5 & 45.5 & 2 & 12 \\
\hline Dhannalal & 9 & 5 & 2 & 8 & 93 & 5 & 45.5 & 3 & 21.5 \\
\hline Anarsingh & 7 & 5 & 2 & 8 & 93 & 6 & 61.5 & 3 & 21.5 \\
\hline Gajrajsingh & 7 & 7 & 1 & 8 & 93 & 6 & 61.5 & 3 & 21.5 \\
\hline Harprasad & 9 & 6 & 5 & 8 & 93 & 6 & 61.5 & 3 & 21.5 \\
\hline Maharajsingh & 6 & 6 & 3 & 8 & 93 & 6 & 61.5 & 3 & 21.5 \\
\hline Shakti singh & 6 & 4 & 2 & 6 & 61.5 & 32 & 35 & 1 & 4 \\
\hline Kamala bai & 9 & 7 & 2 & 8 & 93 & 6 & 61.5 & 3 & 21.5 \\
\hline Raghuveersingh & 9 & 5 & 5 & 8 & 93 & 6 & 61.5 & 3 & 21.5 \\
\hline Vipinsingh & 7 & 5 & 3 & 8 & 93 & 6 & 61.5 & 3 & 21.5 \\
\hline Ashok & 8 & 7 & 1 & 8 & 93 & 6 & 61.5 & 3 & 21.5 \\
\hline Bharat singh & 8 & 6 & 3 & 9 & 103 & 6 & 61.5 & 3 & 21.5 \\
\hline Baboolal & 9 & 4 & 2 & 9 & 103 & 6 & 61.5 & 4 & 32 \\
\hline Parsuram & 9 & 7 & 4 & 9 & 103 & 7 & 79 & 4 & 32 \\
\hline Bhagawansingh & 6 & 5 & 4 & 9 & 103 & 7 & 79 & 4 & 32 \\
\hline Hariram & 7 & 7 & 3 & 9 & 103 & 7 & 79 & 4 & 32 \\
\hline Bhairosingh & 8 & 5 & 1 & 9 & 103 & 7 & 79 & 4 & 32 \\
\hline Radheshyam & 8 & 5 & 3 & 9 & 103 & 7 & 79 & 4 & 32 \\
\hline Saitansingh & 9 & 6 & 2 & 9 & 103 & 7 & 79 & 4 & 32 \\
\hline Lakshminarayan & 6 & 5 & 2 & 9 & 103 & 7 & 79 & 5 & 45.5 \\
\hline Maharajsingh & 7 & 6 & 3 & 9 & 103 & 7 & 79 & 5 & 45.5 \\
\hline Jagannath & 7 & 5 & 3 & 9 & 103 & 7 & 79 & 5 & 45.5 \\
\hline Average & - & & & - & 86.92 & - & 57.21 & - & 19.76 \\
\hline
\end{tabular}


Table.6 Scoring for availability of nearby city in Sayar WUA

\begin{tabular}{|c|c|c|c|c|c|c|c|c|c|}
\hline \multirow[t]{2}{*}{ Farmers name } & \multicolumn{3}{|c|}{$\begin{array}{l}\text { Marks given by the farmers, } \\
\text { for different Irrigation } \\
\text { methods }\end{array}$} & \multicolumn{2}{|c|}{ Sprinkler } & \multicolumn{2}{|c|}{ Border } & \multicolumn{2}{|c|}{ Flood } \\
\hline & Sprinkler & Border & Flood & Obs & Rank & Obs & Rank & Obs & Rank \\
\hline Shakti singh & 5 & 5 & 9 & 2 & 4 & 4 & 27 & 7 & 77.5 \\
\hline Imaratsingh & 3 & 5 & 9 & 2 & 4 & 4 & 27 & 7 & 77.5 \\
\hline Gaindalal & 4 & 4 & 9 & 2 & 4 & 4 & 27 & 7 & 77.5 \\
\hline Ajabsingh & 2 & 5 & 8 & 2 & 4 & 4 & 27 & 7 & 77.5 \\
\hline Ganeshram & 5 & 5 & 7 & 2 & 4 & 4 & 27 & 7 & 77.5 \\
\hline Nandram & 4 & 6 & 7 & 2 & 4 & 4 & 27 & 7 & 77.5 \\
\hline Vishal singh & 4 & 6 & 9 & 3 & 11.5 & 4 & 27 & 7 & 77.5 \\
\hline Sangramsingh & 2 & 4 & 8 & 3 & 11.5 & 4 & 27 & 7 & 77.5 \\
\hline Tularam & 5 & 4 & 8 & 3 & 11.5 & 4 & 27 & 7 & 77.5 \\
\hline Dasharthsingh & 3 & 5 & 9 & 3 & 11.5 & 4 & 27 & 8 & 89 \\
\hline Deewansingh & 5 & 6 & 7 & 3 & 11.5 & 4 & 27 & 8 & 89 \\
\hline Harinarayan & 2 & 5 & 9 & 3 & 11.5 & 5 & 51 & 8 & 89 \\
\hline Bhairosingh & 5 & 6 & 7 & 3 & 11.5 & 5 & 51 & 8 & 89 \\
\hline Shyamlal & 4 & 4 & 9 & 3 & 11.5 & 5 & 51 & 8 & 89 \\
\hline Rupsingh & 4 & 4 & 9 & 4 & 27 & 5 & 51 & 8 & 89 \\
\hline Dhannalal & 2 & 6 & 8 & 4 & 27 & 5 & 51 & 8 & 89 \\
\hline Anarsingh & 3 & 5 & 7 & 4 & 27 & 5 & 51 & 8 & 89 \\
\hline Gajrajsingh & 3 & 5 & 7 & 4 & 27 & 5 & 51 & 8 & 89 \\
\hline Harprasad & 4 & 4 & 8 & 4 & 27 & 5 & 51 & 8 & 89 \\
\hline Maharajsingh & 4 & 5 & 7 & 4 & 27 & 5 & 51 & 8 & 89 \\
\hline Shakti singh & 2 & 4 & 8 & 2 & 4 & 4 & 27 & 7 & 77.5 \\
\hline Kamala bai & 5 & 6 & 9 & 4 & 27 & 5 & 51 & 8 & 89 \\
\hline Raghuveersingh & 2 & 5 & 9 & 4 & 27 & 5 & 51 & 8 & 89 \\
\hline Vipinsingh & 3 & 4 & 8 & 4 & 27 & 5 & 51 & 9 & 102 \\
\hline Ashok & 3 & 5 & 8 & 4 & 27 & 5 & 51 & 9 & 102 \\
\hline Bharat singh & 4 & 5 & 7 & 4 & 27 & 5 & 51 & 9 & 102 \\
\hline Baboolal & 4 & 6 & 9 & 5 & 51 & 5 & 51 & 9 & 102 \\
\hline Parsuram & 2 & 4 & 9 & 5 & 51 & 6 & 68 & 9 & 102 \\
\hline Bhagawansingh & 5 & 5 & 8 & 5 & 51 & 6 & 68 & 9 & 102 \\
\hline Hariram & 3 & 6 & 8 & 5 & 51 & 6 & 68 & 9 & 102 \\
\hline Bhairosingh & 3 & 4 & 7 & 5 & 51 & 6 & 68 & 9 & 102 \\
\hline Radheshyam & 5 & 5 & 7 & 5 & 51 & 6 & 68 & 9 & 102 \\
\hline Saitansingh & 4 & 4 & 8 & 5 & 51 & 6 & 68 & 9 & 102 \\
\hline Lakshminarayan & 4 & 4 & 8 & 5 & 51 & 6 & 68 & 9 & 102 \\
\hline Maharajsingh & 5 & 5 & 8 & 5 & 51 & 6 & 68 & 9 & 102 \\
\hline Jagannath & 5 & 6 & 9 & 5 & 51 & 6 & 68 & 9 & 102 \\
\hline Average & & - & & - & 25.75 & - & 47.25 & - & 0.50 \\
\hline
\end{tabular}


Table.7 Scoring for time saving of irrigation method in command area in Sayar WUA

\begin{tabular}{|c|c|c|c|c|c|c|c|c|c|}
\hline \multirow[t]{2}{*}{ Farmers name } & \multicolumn{3}{|c|}{$\begin{array}{l}\text { Marks given by the farmers, for } \\
\text { different Irrigation methods }\end{array}$} & \multicolumn{2}{|c|}{ Sprinkler } & \multicolumn{2}{|c|}{ Border } & \multicolumn{2}{|c|}{ Flood } \\
\hline & Sprinkler & Border & Flood & Obs & Rank & Obs & Rank & Obs & Rank \\
\hline Shakti singh & 6 & 3 & 3 & 5 & 66.5 & 2 & 5 & 3 & 22 \\
\hline Imaratsingh & 9 & 5 & 5 & 5 & 66.5 & 2 & 5 & 3 & 22 \\
\hline Gaindalal & 8 & 3 & 5 & 5 & 66.5 & 2 & 5 & 3 & 22 \\
\hline Ajabsingh & 9 & 4 & 3 & 5 & 66.5 & 2 & 5 & 3 & 22 \\
\hline Ganeshram & 9 & 2 & 6 & 5 & 66.5 & 2 & 5 & 3 & 22 \\
\hline Nandram & 9 & 5 & 3 & 5 & 66.5 & 2 & 5 & 3 & 22 \\
\hline Vishal singh & 5 & 3 & 3 & 6 & 81.5 & 2 & 5 & 3 & 22 \\
\hline Sangramsingh & 7 & 3 & 4 & 6 & 81.5 & 2 & 5 & 3 & 22 \\
\hline Tularam & 5 & 2 & 4 & 6 & 81.5 & 3 & 22 & 3 & 22 \\
\hline Dasharthsingh & 8 & 4 & 5 & 6 & 81.5 & 3 & 22 & 3 & 22 \\
\hline$\overline{\text { Deewansingh }}$ & 5 & 4 & 5 & 6 & 81.5 & 3 & 22 & 3 & 22 \\
\hline Harinarayan & 9 & 2 & 4 & 6 & 81.5 & 3 & 22 & 3 & 22 \\
\hline Bhairosingh & 7 & 2 & 4 & 6 & 81.5 & 3 & 22 & 4 & 45.5 \\
\hline Shyamlal & 9 & 4 & 5 & 7 & 89 & 3 & 22 & 4 & 45.5 \\
\hline Rupsingh & 5 & 3 & 6 & 7 & 89 & 3 & 22 & 4 & 45.5 \\
\hline Dhannalal & 6 & 3 & 3 & 7 & 89 & 3 & 22 & 4 & 45.5 \\
\hline Anarsingh & 6 & 5 & 3 & 7 & 89 & 3 & 22 & 4 & 45.5 \\
\hline Gajrajsingh & 8 & 4 & 4 & 7 & 89 & 3 & 22 & 4 & 45.5 \\
\hline Harprasad & 9 & 4 & 5 & 8 & 94 & 3 & 22 & 4 & 45.5 \\
\hline Maharajsingh & 7 & 2 & 6 & 8 & 94 & 3 & 22 & 4 & 45.5 \\
\hline Shakti singh & 9 & 2 & 4 & 5 & 66.5 & 2 & 5 & 3 & 22 \\
\hline Kamala bai & 9 & 3 & 3 & 8 & 94 & 4 & 45.5 & 4 & 45.5 \\
\hline Raghuveersingh & 6 & 5 & 3 & 8 & 94 & 4 & 45.5 & 4 & 45.5 \\
\hline Vipinsingh & 5 & 3 & 3 & 8 & 94 & 4 & 45.5 & 4 & 45.5 \\
\hline Ashok & 9 & 2 & 4 & 9 & 102.5 & 4 & 45.5 & 4 & 45.5 \\
\hline Bharat singh & 5 & 3 & 5 & 9 & 102.5 & 4 & 45.5 & 5 & 66.5 \\
\hline Baboolal & 9 & 4 & 4 & 9 & 102.5 & 4 & 45.5 & 5 & 66.5 \\
\hline Parsuram & 8 & 2 & 5 & 9 & 102.5 & 4 & 45.5 & 5 & 66.5 \\
\hline Bhagawansingh & 5 & 4 & 3 & 9 & 102.5 & 4 & 45.5 & 5 & 66.5 \\
\hline Hariram & 6 & 3 & 4 & 9 & 102.5 & 4 & 45.5 & 5 & 66.5 \\
\hline Bhairosingh & 7 & 4 & 3 & 9 & 102.5 & 4 & 45.5 & 5 & 66.5 \\
\hline Radheshyam & 6 & 3 & 4 & 9 & 102.5 & 5 & 66.5 & 5 & 66.5 \\
\hline Saitansingh & 6 & 3 & 3 & 9 & 102.5 & 5 & 66.5 & 5 & 66.5 \\
\hline Lakshminarayan & 8 & 2 & 3 & 9 & 102.5 & 5 & 66.5 & 6 & 81.5 \\
\hline Maharajsingh & 9 & 5 & 4 & 9 & 102.5 & 5 & 66.5 & 6 & 81.5 \\
\hline Jagannath & 7 & 4 & 4 & 9 & 102.5 & 5 & 66.5 & 6 & 81.5 \\
\hline Average & - & & & - & 88.36 & - & 30.46 & - & 44.68 \\
\hline
\end{tabular}


Table.8 Scoring for ease operation of various irrigation methods in Sayar WUA

\begin{tabular}{|c|c|c|c|c|c|c|c|c|c|}
\hline \multirow[t]{2}{*}{ Farmers name } & \multicolumn{3}{|c|}{$\begin{array}{l}\text { Marks given by the farmers, for } \\
\text { different Irrigation methods }\end{array}$} & \multicolumn{2}{|c|}{ Sprinkler } & \multicolumn{2}{|c|}{ Border } & \multicolumn{2}{|c|}{ Flood } \\
\hline & Sprinkler & Border & Flood & Obs & Rank & Obs & Rank & Obs & Rank \\
\hline Shakti singh & 6 & 5 & 2 & 6 & 76.5 & 2 & 8 & 2 & 8 \\
\hline Imaratsingh & 8 & 2 & 3 & 6 & 76.5 & 2 & 8 & 2 & 8 \\
\hline Gaindalal & 9 & 3 & 4 & 6 & 76.5 & 2 & 8 & 2 & 8 \\
\hline Ajabsingh & 9 & 5 & 2 & 6 & 76.5 & 2 & 8 & 2 & 8 \\
\hline Ganeshram & 6 & 2 & 4 & 6 & 76.5 & 2 & 8 & 2 & 8 \\
\hline Nandram & 9 & 3 & 3 & 6 & 76.5 & 3 & 23 & 2 & 8 \\
\hline Vishal singh & 6 & 4 & 3 & 6 & 76.5 & 3 & 23 & 2 & 8 \\
\hline Sangramsingh & 9 & 2 & 4 & 6 & 76.5 & 3 & 23 & 2 & 8 \\
\hline Tularam & 7 & 4 & 2 & 6 & 76.5 & 3 & 23 & 3 & 23 \\
\hline Dasharthsingh & 6 & 4 & 4 & 6 & 76.5 & 3 & 23 & 3 & 23 \\
\hline Deewansingh & 8 & 3 & 4 & 6 & 76.5 & 3 & 23 & 3 & 23 \\
\hline Harinarayan & 9 & 3 & 5 & 6 & 76.5 & 3 & 23 & 3 & 23 \\
\hline Bhairosingh & 7 & 2 & 2 & 7 & 90.5 & 4 & 41.5 & 3 & 23 \\
\hline Shyamlal & 7 & 4 & 3 & 7 & 90.5 & 4 & 41.5 & 3 & 23 \\
\hline Rupsingh & 6 & 6 & 4 & 7 & 90.5 & 4 & 41.5 & 3 & 23 \\
\hline Dhannalal & 8 & 3 & 4 & 7 & 90.5 & 4 & 41.5 & 3 & 23 \\
\hline Anarsingh & 6 & 5 & 2 & 7 & 90.5 & 4 & 41.5 & 4 & 41.5 \\
\hline Gajrajsingh & 6 & 2 & 5 & 7 & 90.5 & 4 & 41.5 & 4 & 41.5 \\
\hline Harprasad & 7 & 4 & 3 & 7 & 90.5 & 4 & 41.5 & 4 & 41.5 \\
\hline Maharajsingh & 7 & 4 & 3 & 7 & 90.5 & 4 & 41.5 & 4 & 41.5 \\
\hline Shakti singh & 7 & 3 & 5 & 6 & 76.5 & 2 & 8 & 2 & 8 \\
\hline Kamala bai & 9 & 5 & 2 & 7 & 90.5 & 4 & 41.5 & 4 & 41.5 \\
\hline Raghuveersingh & 9 & 3 & 4 & 7 & 90.5 & 4 & 41.5 & 4 & 41.5 \\
\hline Vipinsingh & 6 & 6 & 5 & 8 & 98 & 4 & 41.5 & 4 & 41.5 \\
\hline Ashok & 8 & 2 & 2 & 8 & 98 & 5 & 60 & 4 & 41.5 \\
\hline Bharat singh & 7 & 5 & 4 & 8 & 98 & 5 & 60 & 4 & 41.5 \\
\hline Baboolal & 6 & 4 & 2 & 8 & 98 & 5 & 60 & 4 & 41.5 \\
\hline Parsuram & 6 & 6 & 3 & 8 & 98 & 5 & 60 & 4 & 41.5 \\
\hline Bhagawansingh & 7 & 4 & 5 & 9 & 104.5 & 5 & 60 & 5 & 60 \\
\hline Hariram & 9 & 4 & 5 & 9 & 104.5 & 5 & 60 & 5 & 60 \\
\hline Bhairosingh & 6 & 5 & 2 & 9 & 104.5 & 5 & 60 & 5 & 60 \\
\hline Radheshyam & 7 & 4 & 5 & 9 & 104.5 & 6 & 76.5 & 5 & 60 \\
\hline Saitansingh & 6 & 5 & 3 & 9 & 104.5 & 6 & 76.5 & 5 & 60 \\
\hline Lakshminarayan & 6 & 6 & 4 & 9 & 104.5 & 6 & 76.5 & 5 & 60 \\
\hline Maharajsingh & 8 & 6 & 5 & 9 & 104.5 & 6 & 76.5 & 5 & 60 \\
\hline Jagannath & 7 & 4 & 4 & 9 & 104.5 & 6 & 76.5 & 5 & 60 \\
\hline Average & - & & & & 89.60 & - & 40.78 & - & 33.13 \\
\hline
\end{tabular}


Table.9 Scoring for water saving in irrigation methods of Sayar WUA

\begin{tabular}{|c|c|c|c|c|c|c|c|c|c|}
\hline \multirow[t]{2}{*}{ Farmers name } & \multicolumn{3}{|c|}{$\begin{array}{l}\text { Marks given by the farmers, for } \\
\text { different Irrigation methods }\end{array}$} & \multicolumn{2}{|c|}{ Sprinkler } & \multicolumn{2}{|c|}{ Border } & \multicolumn{2}{|c|}{ Flood } \\
\hline & Sprinkler & Border & Flood & Obs & Rank & Obs & Rank & Obs & Rank \\
\hline Shakti singh & 8 & 6 & 4 & 6 & 62 & 4 & 32 & 2 & 8 \\
\hline Imaratsingh & 6 & 4 & 2 & 6 & 62 & 4 & 32 & 2 & 8 \\
\hline Gaindalal & 8 & 6 & 2 & 6 & 62 & 4 & 32 & 2 & 8 \\
\hline Ajabsingh & 7 & 6 & 3 & 6 & 62 & 4 & 32 & 2 & 8 \\
\hline Ganeshram & 9 & 5 & 4 & 7 & 80 & 4 & 32 & 2 & 8 \\
\hline Nandram & 9 & 4 & 3 & 7 & 80 & 5 & 45.5 & 2 & 8 \\
\hline Vishal singh & 6 & 7 & 4 & 7 & 80 & 5 & 45.5 & 2 & 8 \\
\hline Sangramsingh & 7 & 7 & 3 & 7 & 80 & 5 & 45.5 & 2 & 8 \\
\hline Tularam & 8 & 5 & 2 & 7 & 80 & 5 & 45.5 & 2 & 8 \\
\hline Dasharthsingh & 7 & 4 & 2 & 7 & 80 & 5 & 45.5 & 2 & 8 \\
\hline Deewansingh & 9 & 7 & 3 & 7 & 80 & 5 & 45.5 & 2 & 8 \\
\hline Harinarayan & 7 & 5 & 2 & 7 & 80 & 5 & 45.5 & 2 & 8 \\
\hline Bhairosingh & 9 & 6 & 3 & 7 & 80 & 5 & 45.5 & 2 & 8 \\
\hline Shyamlal & 6 & 6 & 4 & 7 & 80 & 5 & 45.5 & 2 & 8 \\
\hline Rupsingh & 9 & 7 & 3 & 7 & 80 & 5 & 45.5 & 3 & 20.5 \\
\hline Dhannalal & 9 & 5 & 2 & 8 & 93.5 & 6 & 62 & 3 & 20.5 \\
\hline Anarsingh & 7 & 7 & 2 & 8 & 93.5 & 6 & 62 & 3 & 20.5 \\
\hline Gajrajsingh & 9 & 5 & 2 & 8 & 93.5 & 6 & 62 & 3 & 20.5 \\
\hline Harprasad & 8 & 4 & 4 & 8 & 93.5 & 6 & 62 & 3 & 20.5 \\
\hline Maharajsingh & 8 & 6 & 5 & 8 & 93.5 & 6 & 62 & 3 & 20.5 \\
\hline Shakti singh & 7 & 5 & 5 & 6 & 62 & 4 & 32 & 2 & 8 \\
\hline Kamala bai & 6 & 7 & 4 & 8 & 93.5 & 6 & 62 & 3 & 20.5 \\
\hline Raghuveersingh & 9 & 5 & 2 & 8 & 93.5 & 6 & 62 & 3 & 20.5 \\
\hline Vipinsingh & 7 & 6 & 2 & 8 & 93.5 & 6 & 62 & 3 & 20.5 \\
\hline Ashok & 8 & 6 & 3 & 8 & 93.5 & 6 & 62 & 3 & 20.5 \\
\hline Bharat singh & 7 & 6 & 2 & 8 & 93.5 & 6 & 62 & 4 & 32 \\
\hline Baboolal & 7 & 5 & 3 & 9 & 103.5 & 6 & 62 & 4 & 32 \\
\hline Parsuram & 9 & 5 & 2 & 9 & 103.5 & 6 & 62 & 4 & 32 \\
\hline Bhagawansingh & 8 & 6 & 4 & 9 & 103.5 & 6 & 62 & 4 & 32 \\
\hline Hariram & 8 & 4 & 2 & 9 & 103.5 & 6 & 62 & 4 & 32 \\
\hline Bhairosingh & 6 & 6 & 3 & 9 & 103.5 & 7 & 70 & 4 & 32 \\
\hline Radheshyam & 7 & 5 & 2 & 9 & 103.5 & 7 & 70 & 4 & 32 \\
\hline Saitansingh & 8 & 6 & 2 & 9 & 103.5 & 7 & 70 & 5 & 45.5 \\
\hline Lakshminarayan & 7 & 4 & 5 & 9 & 103.5 & 7 & 70 & 5 & 45.5 \\
\hline Maharajsingh & 9 & 6 & 3 & 9 & 103.5 & 7 & 70 & 5 & 45.5 \\
\hline Jagannath & 8 & 6 & 5 & 9 & 103.5 & 7 & 70 & 5 & 45.5 \\
\hline Average & - & & & - & 87.78 & - & $\mathbf{5 3 . 7 5}$ & - & 20.31 \\
\hline
\end{tabular}


Table.10 Scoring for high income irrigation method in command area in Sayar WUA

\begin{tabular}{|c|c|c|c|c|c|c|c|c|c|}
\hline \multirow[t]{2}{*}{ Farmers name } & \multicolumn{3}{|c|}{$\begin{array}{l}\text { Marks given by the farmers, for } \\
\text { different Irrigation methods }\end{array}$} & \multicolumn{2}{|c|}{ Sprinkler } & \multicolumn{2}{|c|}{ Border } & \multicolumn{2}{|c|}{ Flood } \\
\hline & Sprinkler & Border & Flood & Obs & Rank & Obs & Rank & Obs & Rank \\
\hline Shakti singh & 8 & 5 & 2 & 5 & 61.5 & 3 & 17.5 & 2 & 4 \\
\hline Imaratsingh & 7 & 4 & 3 & 5 & 61.5 & 3 & 17.5 & 2 & 4 \\
\hline Gaindalal & 6 & 3 & 4 & 5 & 61.5 & 3 & 17.5 & 2 & 4 \\
\hline Ajabsingh & 7 & 5 & 2 & 5 & 61.5 & 3 & 17.5 & 2 & 4 \\
\hline Ganeshram & 5 & 4 & 5 & 5 & 61.5 & 3 & 17.5 & 2 & 4 \\
\hline Nandram & 6 & 3 & 5 & 5 & 61.5 & 3 & 17.5 & 2 & 4 \\
\hline Vishal singh & 7 & 5 & 3 & 5 & 61.5 & 3 & 17.5 & 3 & 17.5 \\
\hline Sangramsingh & 8 & 4 & 4 & 5 & 61.5 & 3 & 17.5 & 3 & 17.5 \\
\hline Tularam & 5 & 3 & 3 & 5 & 61.5 & 3 & 17.5 & 3 & 17.5 \\
\hline Dasharthsingh & 6 & 6 & 4 & 6 & 82.5 & 4 & 38 & 3 & 17.5 \\
\hline Deewansingh & 7 & 3 & 2 & 6 & 82.5 & 4 & 38 & 3 & 17.5 \\
\hline Harinarayan & 8 & 6 & 5 & 6 & 82.5 & 4 & 38 & 3 & 17.5 \\
\hline Bhairosingh & 5 & 4 & 4 & 6 & 82.5 & 4 & 38 & 3 & 17.5 \\
\hline Shyamlal & 6 & 6 & 4 & 6 & 82.5 & 4 & 38 & 3 & 17.5 \\
\hline Rupsingh & 7 & 3 & 3 & 6 & 82.5 & 4 & 38 & 3 & 17.5 \\
\hline Dhannalal & 7 & 4 & 3 & 6 & 82.5 & 4 & 38 & 3 & 17.5 \\
\hline Anarsingh & 5 & 6 & 5 & 6 & 82.5 & 4 & 38 & 4 & 38 \\
\hline Gajrajsingh & 8 & 3 & 2 & 7 & 95 & 4 & 38 & 4 & 38 \\
\hline Harprasad & 6 & 5 & 4 & 7 & 95 & 5 & 61.5 & 4 & 38 \\
\hline Maharajsingh & 7 & 6 & 5 & 7 & 95 & 5 & 61.5 & 4 & 38 \\
\hline Shakti singh & 5 & 3 & 4 & 5 & 61.5 & 3 & 17.5 & 2 & 4 \\
\hline Kamala bai & 5 & 4 & 3 & 7 & 95 & 5 & 61.5 & 4 & 38 \\
\hline Raghuveersingh & 7 & 6 & 4 & 7 & 95 & 5 & 61.5 & 4 & 38 \\
\hline Vipinsingh & 6 & 4 & 2 & 7 & 95 & 5 & 61.5 & 4 & 38 \\
\hline Ashok & 5 & 3 & 5 & 7 & 95 & 5 & 61.5 & 4 & 38 \\
\hline Bharat singh & 8 & 5 & 3 & 7 & 95 & 5 & 61.5 & 4 & 38 \\
\hline Baboolal & 6 & 5 & 4 & 7 & 95 & 5 & 61.5 & 4 & 38 \\
\hline Parsuram & 5 & 3 & 3 & 8 & 104 & 5 & 61.5 & 4 & 38 \\
\hline Bhagawansingh & 8 & 5 & 5 & 8 & 104 & 6 & 82.5 & 4 & 38 \\
\hline Hariram & 6 & 4 & 2 & 8 & 104 & 6 & 82.5 & 5 & 61.5 \\
\hline Bhairosingh & 7 & 6 & 3 & 8 & 104 & 6 & 82.5 & 5 & 61.5 \\
\hline Radheshyam & 5 & 3 & 4 & 8 & 104 & 6 & 82.5 & 5 & 61.5 \\
\hline Saitansingh & 8 & 4 & 2 & 8 & 104 & 6 & 82.5 & 5 & 61.5 \\
\hline Lakshminarayan & 8 & 5 & 4 & 8 & 104 & 6 & 82.5 & 5 & 61.5 \\
\hline Maharajsingh & 5 & 6 & 3 & 8 & 104 & 6 & 82.5 & 5 & 61.5 \\
\hline Jagannath & 8 & 5 & 4 & 8 & 104 & 6 & 82.5 & 5 & 61.5 \\
\hline Average & - & - & - & & 85.17 & - & 48.07 & - & 30.26 \\
\hline
\end{tabular}


The Chi square test value was 78.72 which was larger than the tabulated value 5.99 and 9.21 on 2 degree of freedom at $5 \%$ and $1 \%$ level of significance. Thus the null hypothesis was rejected and found that there was significant difference in values was observed among sprinkler irrigation, border irrigation and flood irrigation methods.

The Chi square test value was 83.11 which was larger than the tabulated value 5.99 and 9.21 on 2 degree of freedom at $5 \%$ and $1 \%$ level of significance. Thus the null hypothesis was rejected and concluded that there were significant difference among sprinkler irrigation, border irrigation and flood irrigation methods as for as scoring for control and management of water in command area of Sayar water user association was concerned.

The calculated Chi square test value was 79.82 which was larger than the tabulated value 5.99 and 9.21 on 2 degree of freedom at $5 \%$ and $1 \%$ level of significance.

Thus the null hypothesis was rejected. The Chi square test value was 66.82 which was larger than the tabulated value 5.99 and 9.21 on 2 degree of freedom at $5 \%$ and $1 \%$ level of significance. It was concluded that time of saving matters among sprinkler irrigation, border irrigation and flood irrigation system.

The Chi square test value was 68.88 which was larger than the tabulated value 5.99 and 9.21 on 2 degree of freedom at $5 \%$ and $1 \%$ level of significance. Thus the null hypothesis was rejected and concluded that ease of operation of irrigation methods is very important for farmers of Sayar water user association.

The Chi square test value was 83.53 which was larger than the tabulated value 5.99 and 9.21 on 2 degree of freedom at $5 \%$ and $1 \%$ level of significance. Thus the null hypothesis was rejected and concluded that there was significant difference among sprinkler irrigation, border irrigation and flood irrigation methods as for as water saving in irrigation methods of Sayar WUA is concerned.

The Chi square test value was 57.58 which was larger than the tabulated value 5.99 and 9.21 on 2 degree of freedom at $5 \%$ and $1 \%$ level of significance. Thus the null hypothesis was rejected and concluded that there were significant difference among sprinkler irrigation, border irrigation and flood irrigation methods.

Significant difference was observed in adoption of sprinkler irrigation system, border irrigation system and flood irrigation system by farmers of the right bank canal command area when the score matrix for Labour requirement, Cost, Suitability of irrigation, Control and management of water, Availability of nearby city, Time saving, Ease operation, Water saving, High income was analyzed through Chi-square test. Similar study was also conducted for water user association namely Sarchampa, Ucher, Medaki and Neemkheda and the similar result was found (Table 1-10).

Characters like awareness of farmers, technical knowledge, attitude, willingness of adoption and purchasing capacity of farmers were consider, analyzed and score matrix was prepared for each character. The Chi square test was applied and it was concluded that type of farmers and their awareness, technical knowledge, attitude, willingness of adoption and purchasing capacity of farmers dependent on each other that is the large farmer could have the different awareness, technical knowledge, attitude, willingness of adoption and purchasing capacity than marginal, small and medium farmers. 


\section{Acknowledgments}

I would like to extend my sincere thanks to my advisor, Dr. S.K. Pyasi, for extending knowledge, guidance, freedom during the course this project.

\section{References}

Anonymous, 2016. Annual Report of Samrat Ashok Sagar Project, Irrigation department Vidisha Madhya Pradesh, 123.

Ghosh, S., Kannan, K., Singh, R., and Kundu, D. K. 2002. Socio-economic Profile and Cropping Pattern in Canal Command Area in Khurda District of Orissa. Indian Journal of Extension Education. 38 (1\&2): 99-103.

Pereira, L.S., Cordery, I., and Iacovides, I. 2012. Improved indicators of water use performance and productivity for sustainable water conservation and saving. Agric. Water Manag, 108, 3951.

Perry, C. J., and Narayanamurthy, S. G. 1998. Farmer Response to Rationed and Uncertain Irrigation Supplies, IWMI Research Report 24, pp 1-23

Pute, W., Hao, F., Wenquan, N., Jianen, G., Dingsheng, J., Youke, W., Xingke, F., and Peng, Q. 2003.Analysis of developmental tendency of water distribution and water-saving strategies. Trans. Chin. Soc. Agric. Engg. 19, 1-6.
Rathod, S. A., and Shah, S. D. 2013. Design Principales and Consideration for Pressurized Irrigation System, A Case Study, 2(3), 137-13

Sevendsen, M., and Small, L. E. 1990.Farmers' Perspective on Irrigation Performance. Irrigation and Drainage system, 4: 385-402.

Shibiao, F., Renfu, Jia., Wenrong, Tu., and Zhilin, Sun. 2017. Assessing Factors Driving the Change of Irrigation WaterUse Efficiency in China Based on Geographical Features, open access journal on water science and technology, 1-17

Singh, R., and Kundu, D. K., and Kannan, K.2003. Crop Diversification through Land Modification in Canal Irrigation Command of Eastern India. SAARK Journal of Agriculture. 1(1): 99-104.

Tanwar, B. S. 1998. Water Management though People's Participation in India. The Tenth ICID Afro-Asian Regeonal Conference on Irrigation and Drainage, Denpasar, Bali Indonesia, C8: 9-11.

Turkar, R., 2014. Adaptive Irrigation Management Planning - A Farmer's perspective. M. Tech Thesis JNKVV Jaqbalpur, p 28.

Wang, G.F., Chen, J.C., Wu, F., Li, Z.H. 2015. An integrated analysis of agricultural water-use efficiency: A case study in the Heihe River Basin in Northwest China. Phys. Chem. Earth, $89,3-9$.

\section{How to cite this article:}

Shiv Singh Basediya, S.K. Pyasi and Shrivastava, R.N. 2018. A Study on Farmer Response for Technical Intervention in Canal Command Area of Samrat Ashok Sagar Project. Int.J.Curr.Microbiol.App.Sci. 7(03): 3387-3401. doi: https://doi.org/10.20546/ijcmas.2018.703.391 